\title{
Energy Crisis and Nuclear Fusion Power
}

\author{
Hari K.C. \\ Department of Physics, Prithvi Narayan Campus, Pokhara
}

\begin{abstract}
Due to the rapid industrialisation and luxurious life style of mankind all of the non renewable sources of energy are going to finish in near future. To address energy crisis problem it is necessary to search or invent long lasting new sources of energy which could run the world for long term. Here, in this article a attempt has been made to discuss a new source of energy called nuclear fusion process. Several methods made to produce energy from nuclear fusion process are tried to discuss here. Fusion power offers the prospect of an almost inexhaustible source of energy for future generations, but it also presents so far insurmountable scientific and engineering challenges. The main hope is centered on tokamak reactors which confine a deuterium-tritium plasma magnetically.
\end{abstract}

Keywords: energy crisis, fusion, plasma, confinement, tokamak.

\section{INTRODUCTION:}

The continuous use of non-renewable sources of energy causes the serious problem of energy Scarcity. Such an energy scarcity is called energy crisis. Thus the energy crisis is a situation in which there will be increasing demand and decreasing production of fossil fuels. The human being has to face several difficulties and the human activities will be slowed down due to energy crisis.

Energy is the most important part of our life which is obtained from various sources like coal natural gases, lp gases, water, wood, etc. due to the growth of modern civilization and increasing population more energy has been used up. Thus there is always an increasing demands of energy throughout the world. The petroleum products, coal etc are being finished, forests are being reduced. Since the demand for energy is increasing rapidly, the present sources of energy cannot last for a long time. So there is a need of searching a reliable alternative source of energy to fulfill the excess demand of energy. The aim of the article here is to discuss the nuclear fusion process. Controlled thermonuclear fusion process can fulfill the demand of energy throughout the world.

A process in which two or more lighter atomic nuclei fuse to form a heavier nucleus. In doing so they release comparatively large amount of energy. For example isotopes of hydrogen, duterium and tritium fuse together to form helium and neutron with release of energy.
This process is most important for the production of energy. Due to release of large amount of energy and easier availability of deuterium this process is supposed to be a reliable process for the energy production in earth. Energy released during the fusion of deuterium and tritium is thousand times greater than fission of uranium and billions times the burning of coal.

\section{MECHANISM}

Fusion happens when two (or more) nuclei come close enough for the strong nuclear force to exceed the electrostatic force and pull them together. This process takes light nuclei and forms a heavier one, through a nuclear reaction. For nuclei lighter than iron-56 this is exothormic and releases energy. For nuclei heavier than iron-56 this is endothormic and requires an external source of energy. Hence, nuclei smaller than iron-56 are more likely to fuse while those heavier than iron-56 are more likely to break apart.

To fuse, nuclei must overcome the repulsive Coulomb force. This is a force caused by the nuclei containing positively charged protons that repel via the electromagnetic force. To overcome this coulomb barrier, the atoms must have a high kinetic energy. There are several ways of doing this, including heating or acceleration. Once an atom is heated above its ionisation energy, its electrons are stripped away, leaving just the bare nucleus: the ion .Most fusion experiments use a hot cloud of ions 
and electrons. This cloud is known as a plasma. Most fusion reactions produce neutrons, which can be detected and degrade materials.

Theoretically, any atom could be fused, if enough pressure and temperature was applied.-Mankind has studied many high energy fusion reactions, using particles beams. These are fired at a target. For a power plant, however, we are currently limited to only the light elements. Hydrogen is ideal: because of its small charge, it is the easiest atom to fuse. This reaction produces Helium.

Fusion power research seeks the commercial production of net usable power from a fusion source. The primary problem is confining the fusion reaction so it continues using its own energy. Leading designs for controlled fusion research use inertial or magnetic confinement of a plasma. Both approaches are still under development and are years away from commercial operation.

For fusion fuel different isotopes of hydrogen must be heated to extreme temperatures of the order of 100 million degrees Celsius, and must be kept dense enough, and confined for long enough, to allow the nuclei to fuse. In the Sun and stars massive gravitational forces create the right conditions for fusion. Fusion powers the Sun and stars as hydrogen isotopes fuse together to form helium, and matter is converted into energy. Hydrogen, heated to very high temperatures changes from a gas to a plasma in which the negatively-charged electrons are separated from the positively-charged atomic nuclei (ions). Normally, fusion is not possible because the strongly repulsive electrostatic forces between the positively charged nuclei prevent them from getting close enough together to collide and for fusion to occur. However, if the conditions are such that the attractive nuclear forces dominants the repulsive electrostatic forces between the nuclei, they can fuse together. Such conditions can occur when the temperature increases, causing the ions to move faster and eventually reach speeds high enough to bring the ions close enough together. The nuclei can then fuse, causing a release of energy.

In Earth natural condition to sustain nuclear fission is much harder to achieve. In the Sun, massive gravitational forces create the right conditions for fusion. The aim of the controlled fusion research program is to achieve 'ignition', which occurs when enough fusion reactions take place for the process to become self-sustaining, with fresh fuel then being added to continue it. Once ignition is achieved, there is net energy yield - about four times as much as with nuclear fission.

Initially fusion research in the USA and USSR was linked to atomic weapons development, Following a breakthrough at the Soviet tokamak, fusion research became 'big science' in the 1970s. Today, many countries are taking take part in fusion research to some extent, led by the European Union, the USA, Russia and Japan. Vigorous programs also underway in China, Brazil, Canada, and Korea. But the cost and complexity of the devices involved increased to the point where international co-operation was the only way forward.

With current technology, the reaction most readily feasible is between the nuclei of the two heavy forms (isotopes) of hydrogen - deuterium (D) and tritium (T). Each D-T fusion event releases $17.6 \mathrm{MeV}$ (2.8 x $10^{-12}$ joule, compared with $200 \mathrm{MeV}$ for a U-235 fission). Deuterium occurs naturally in seawater (30 grams per cubic metre), which makes it very abundant relative to other energy resources. Tritium occurs naturally only in trace quantities (produced by cosmic rays) and is radioactive. Usable quantities can be made in a conventional nuclear reactor, or in the present context, bred in a fusion system from lithium

Fusion powers the Sun and stars as hydrogen atoms fuse together to form helium, and matter is converted into energy. Hydrogen, heated to very high temperatures changes from a gas to a plasma in which the negatively-charged electrons are separated from the positively-charged atomic nuclei 
(ions). Normally, fusion is not possible because the strongly repulsive electrostatic forces between the positively charged nuclei prevent them from getting close enough together to collide and for fusion to occur. However, if the conditions are such that the nuclei can overcome the electrostatic forces to the extent that they can come within a very close range of each other, then the attractive nuclear force (which binds protons and neutrons together in atomic nuclei) between the nuclei will outweigh the repulsive (electrostatic) force, allowing the nuclei to fuse together. Such conditions can occur when the temperature increases, causing the ions to move faster and eventually reach speeds high enough to bring the ions close enough together. The nuclei can then fuse, causing a release of energy.

\section{FUSION TECHNOLOGY}

In a fusion reactor, the concept is that neutrons generated from the D-T fusion reaction will be absorbed in a blanket containing lithium which surrounds the core. The lithium is then transformed into tritium (which is used to fuel the reactor) and helium. The blanket must be thick enough (about 1 metre) to slow down the high-energy (14 MeV) neutrons. The kinetic energy of the neutrons is absorbed by the blanket, causing it to heat up. The heat energy is collected by the coolant (water, helium or Li-Pb eutectic) flowing through the blanket and, in a fusion power plant, this energy will be used to generate electricity by conventional methods. If insufficient tritium is produced, some supplementary source must be employed such as using a fission reactor to irradiate heavy water or lithium with neutrons, and extraneous tritium creates difficulties with handling, storage and transport.

The difficulty has been to develop a device that can heat the D-T fuel to a high enough temperature and confine it long enough so that more energy is released through fusion reactions than is used to get the reaction going. While the $\mathrm{D}-\mathrm{T}$ reaction is the main focus of attention, long-term hopes are for a D-D reaction, but this requires much higher temperatures.
At present, two main experimental approaches are being studied: inertial confinement and magnetic confinement. The first involves compressing a small pellet containing fusion fuel to extremely high densities using strong lasers or particle beams. The second method uses strong magnetic fields to contain the hot plasma

\section{Inertial confinement fusion}

In inertial confinement fusion, which is a newer line of research, laser or ion beams are focused very precisely onto the surface of a target, which is a pellet of D-T fuel, a few millimetres in diameter. This heats the outer layer of the material, which explodes outwards generating an inward-moving compression front or implosion that compresses and heats the inner layers of material. The core of the fuel may be compressed to one thousand times its liquid density, resulting in conditions where fusion can occur. The energy released then would heat the surrounding fuel, which may also undergo fusion leading to a chain reaction (known as ignition) as the reaction spreads outwards through the fuel. The time required for these reactions to occur is limited by the inertia of the fuel (hence the name), but is less than a microsecond. So far, most inertial confinement work has involved lasers.

Recent work at Osaka University's Institue of Laser Engineering in Japan suggests that ignition may be achieved at lower temperature with a second very intense laser pulse guided through a millimetre-high gold cone into the compressed fuel, and timed to coincide with the peak compression. This technique, known as 'fast ignition', means that fuel compression is separated from hot spot generation with ignition, making the process more practical.

\section{Magnetic confinement fusion}

In magnetic confinement fusion, hundreds of cubic metres of D-T plasma at a density of less than a milligram per cubic metre are confined by a magnetic field at a few atmospheres pressure and heated to fusion temperature. 
Magnetic fields are ideal for confining a plasma because the electrical charges on the separated ions and electrons mean that they follow the magnetic field lines. The aim is to prevent the particles from coming into contact with the reactor walls as this will dissipate their heat and slow them down. The most effective magnetic configuration is toroidal, shaped like a doughnut, in which the magnetic field is curved around to form a closed loop. For proper confinement, this toroidal field must have superimposed upon it a perpendicular field component (a poloidal field). The result is a magnetic field with force lines following spiral (helical) paths that confine and control the plasma.

There are several types of toroidal confinement system, the most important one being tokamaks. In a tokamak, the toroidal field is created by a series of coils evenly spaced around the torus-shaped reactor, and the poloidal field is created by a system of horizontal coils outside the toroidal magnet structure. A strong electric current is induced in the plasma using a central solenoid, and this induced current also contributes to the poloidal field. In a stellarator, the helical lines of force are produced by a series of coils which may themselves be helical in shape. In tokamaks the current flowing through the plasma also serves to heat it to a temperature of about 10 million degrees Celsius. Beyond that, additional heating systems are needed to achieve the temperatures necessary for fusion.

The tokamak (torus-shaped magnetic chamber) was designed in 1951 by Soviet physicists Lev Artsimovich, Andrei Sakharov and Igor Tamm. It operates within limited parameters outside which sudden losses of energy disruptions can occur. This can cause major thermal and mechanical stresses to the structure and walls. Eventhough it is considered as the most promising design and research is continuing on various tokamaks around the world.

\section{FUSION RESEARCH}

Large numbers of efforts have been made since the 1970s, still commercial deployment of fusion power has is still far away. Many breakthroughs have been made, particularly in recent years, and there are a number of major projects under development that may bring research to the point where fusion power can be used commertially.

Several tokamaks have been built, including the Joint European Torus (JET), the tokamak fusion test reactor (TFTR) at Princeton in U.S.A., Korean superconducting tokamak reactor ( K-STAR). The ITER (International Thermonuclear Experimental Reactor) project currently under construction in Cadarache, France will be the largest tokamak.

\section{JET}

In 1978, the European Community launched the Joint European Torus (JET) project in the UK. JET is the largest tokamak operating in the world today. It has the facilities to use D-T fuel.JET produced its first plasma in 1983, and became the first experiment to produce controlled fusion power in November 1991. JET is a key device in preparations for ITER. It has been significantly upgraded in recent years to test ITER plasma physics and engineering systems.

\section{TFTR}

In the USA, the Tokamak Fusion Test Reactor(TFTR) operated at the Princeton Plasma Physics Laboratory (PPPL) from 1982 to 1997. In December 1993, TFTR became the first magnetic fusion device to perform extensive experiments with plasmas composed of D-T. The following year TFTR produced 10.7 MW of controlled fusion power - a record at that time. TFTR set other records, including the achievement of a plasma temperature of 510 million degrees centigrade in 1995. However, it did not achieve its goal of break-even fusion energy (where the energy input required is no greater than the amount of fusion energy produced), but achieved all of its hardware design goals, thus making substantial contributions to the development of ITER.

\section{K-DEMO}

In collaboration with the US Department of Energy's Princeton Plasma Physics Laboratory (PPPL) in New Jersey and South Korea's National Fusion Research Institute K-DEMO is intended to be the next step 
toward commercial reactors from ITER, and would be the first plant to actually contribute power to an electric grid. Its aim is to generate some 1 billion watts of power a much greater output than ITER's goal of producing 500 megawatts by the 2020 s.

\section{ITER}

In order to generate energy in international level scientists are working hard for next generation tokamak, a dream project ITER (International Thermonuclear Experimental Reactor). Seven parties International Thermonuclear Experimental Reactor. ITER is based on the 'tokamak' concept of magnetic confinement.

In November 2006, the seven members India, China, Japan, Russia, South Korea, the USA and the European Union signed the ITER implementing agreement. Site preparation works at Cadarache commenced in January 2007. First concrete for the buildings was poured in December 2013. Experiments were due to begin in 2020, when producing plasma first time. The first D-T plasma is not expected until 2026. The vacuum vessel will be $19 \mathrm{~m}$ across and $11 \mathrm{~m}$ high, and weigh more than 5000 tonnes.

The goal of ITER is to operate at $500 \mathrm{MW}$ with less than $50 \mathrm{MW}$ of input power, a tenfold energy gain. No electricity will be generated at ITER.

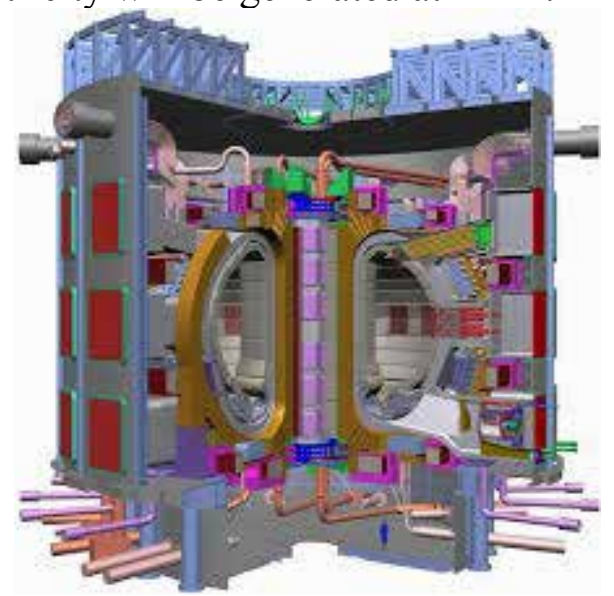

Fig: Early phase of ITER.

A demonstration Power Plant, known as Demo, is expected to demonstrate large-scale production of electrical power on a continual basis. The conceptual design of Demo is expected to be completed by 2017, with construction beginning in around 2024. The goal of Demo is to generate a twenty five times more energy. The first phase of operation commencing from 2033.

\section{NUCLEAR WASTE (HAZARDS)}

Although fusion does not generate harmful gases etc. and long-lived radioactive products, there would a short- to medium-term radioactive waste problem due to activation of the structural materials. Some component materials will become radioactive during the lifetime of a reactor, due to bombardment with high-energy neutrons, and will eventually become radioactive waste. However, the long-term radio toxicity of the fusion wastes would be considerably lower than that from actinides in used fission fuel, and the activation product wastes would be handled in much the same way as those from fission reactors.

There are also other the possibility of release of tritium into the environment. It is radioactive and very difficult to contain since it can penetrate concrete, rubber and some grades of steel. As an isotope of hydrogen, it is easily incorporated into water, making the water itself weakly radioactive. The presence of tritium remains a threat to health for about nearly a hundred years after it is created. It can be inhaled, absorbed through the skin or ingested. Inhaled tritium spreads throughout the soft tissues and tritiated water mixes quickly with all the water in the body. Although there is only a small inventory of tritium in a fusion reactor - a few grams - each could conceivably release significant quantities of tritium during operation through routine leaks, assuming the best containment systems. An accident could release even more. This is one reason why long-term hopes are for the deuterium-deuterium fusion process, dispensing with tritium.

\section{CONCLUSION:}

The use of fusion power plants could substantially reduce the environmental impacts of increasing world electricity demands since, like nuclear fission power, they would not contribute to acid rain or 
the greenhouse effect. Fusion power could easily satisfy the energy needs associated with continued economic growth, given the ready availability of fuels. There would be no danger of a runaway fusion reaction as this is intrinsically impossible and any malfunction would result in a rapid shutdown of the plant. Fusion power clearly has much to offer when the technology is eventually developed; the problems associated with it also need to be addressed if it is to become a widely used future energy source.
REFERENCES:

1. Chen, Francis F. (1984) Plasma, Physics and Controlled Fusion, Plenum Press.

2. Weston M Stacey (2005) Fusion Plasma Physics, Wiley-VCH.

3. Wikipedia www.wikipedia.org

4. WNA www.wna.org

5. ITER Www.iter.org

6. JET www.jet.efda.org 